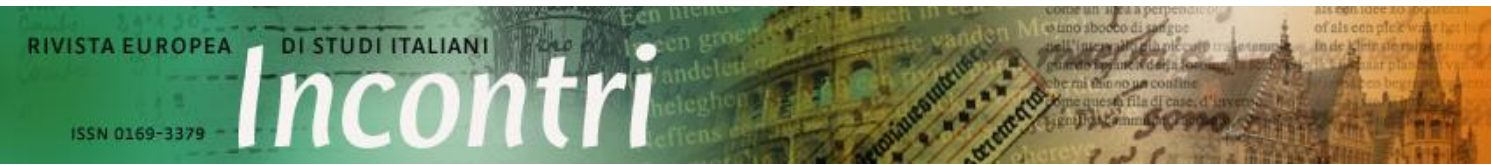

Anno 36, 2021 / Fascicolo 1 / p. 45-59 - www.rivista-incontri.nl - http://doi.org/10.18352/inc11005

(c) The author(s) - Content is licensed under a Creative Commons Attribution 3.0 Unported License Publisher: Werkgroep Italië Studies, supported by openjournals.nl

\title{
Lo spaesamento delle viaggiatrici europee al nord Straniamenti odeporici
}

\author{
Ellen Patat
}

Introduzione

Nel corso dell'Ottocento si assiste a un mutamento delle condizioni di vita e dei trasporti che, di fatto, modificano e sradicano la consueta fissità femminile portando alcune donne al di fuori dei confini domestici. Indubbiamente, quando a viaggiare è una donna, si innescano dei meccanismi di valutazione diversi rispetto alla controparte maschile ${ }^{1}$ in quanto le dinamiche che sottendono alla mobilità femminile di questo frangente storico sono ancora strettamente legate in primis alle norme sociali di stampo patriarcale, che tingono l'esperienza concreta di tratti simbolici; la mera distanza geografica inquadra distanze ben più intime: l'erranza femminile pone, infatti, le viaggiatrici fuori dalla norma, fuori anche dalla riconosciuta dimensione d'accettazione e percezione dei valori e del corpo stesso.

La motilità, che spesso si riverbera nella vita del soggetto viaggiante come un sentimento di spaesamento, è il cardine dell'odeporia, genere che, prendendo il via dall'atavica esigenza conoscitiva dell'essere umano, porta il/la viaggiat-ore/trice a esplorare l'Altrove e l'Altro fuori dalla sua quotidianità. Si va così creando un'intensa dialettica tra la soggettività faziosa e la decostruzione della realtà che essa compie: l'estraneità si trasforma lasciando spazio all'approvazione o al riconoscimento degli altri o di sé stessi.

Il corpus del presente saggio è composto dalle narrazioni odeporiche di quattro viaggiatrici europee che hanno sperimentato e descritto il grande nord europeo ${ }^{2}$ dalla seconda metà dell'Ottocento: in ordine cronologico, Ida Laura Pfeiffer con Visit to Iceland and Scandinavia North ${ }^{3}$ (1853), Carla Serena e il suo Mon voyage: souvenirs

\footnotetext{
${ }^{1}$ Cfr. M. Morris \& L. O’Connor, The Virago Book of Women Travellers, London, Virago, 1996, p. XVII.

2 Meno esotico dell'Oriente, che aveva raccolto così tanta curiosità e dato vita ad altrettanti resoconti, il Nord Europa diventa meta di un crescente interesse; negli studi accademici, tale attenzione si manifesta tardi in raccolte quali J. Hill, White Horizon: The Arctic in the Nineteenth-Century British Imagination, Albany, State University of New York Press, 2008 oppure A. Ryall, J. Schimanski \& H.H. Waerp, Arctic Discourses, Cambridge, Cambridge Scholars Publishing, 2010. Resoconti e riferimenti sulla Scandinavia, in particolare sulla gamle, ossia 'vecchia', Norvegia, vengono riportati in K. Walchester, Gamle Norge and Nineteenth-Century British Women Travellers in Norway, London-New York, Anthem Press, 2014. 'Il Grande Nord quello con la maiuscola', soggetto anch'esso, così come l'Oriente, alla rielaborazione delle culture di matrice europea, 'evoca generalmente l'idea di un luogo di frontiera dai connotati estremi e disumani’ (E. Camanni, Il Grande Libro del Ghiaccio, Bari, Laterza, 2020, cap. VIII [Kindle]).

${ }^{3}$ I. Pfeiffer, Visit to Iceland and Scandinavian North, London, Ingram, Cooke, and Co., 1853. Nell'ambito del progetto Gutenberg è possibile trovare la trascrizione della traduzione dal tedesco della seconda edizione del 1853 (https://gutenberg.unblockit.win/files/1894/1894-h/1894-h.htm, 18 aprile 2020) utilizzata nel presente saggio in conversione pdf dell'ebook [\#1894 - 07.05.2007]. Il testo originale del 1846 in tedesco - I. Pfeiffer, Reise nach dem skandinavischen Norden und der Insel Island, Leipzig,
} 
personnels. De la Baltique à la Mer Caspienne 4 (1881), Mrs Alec-Tweedie con A girl's ride in Iceland ${ }^{5}$ (1889), e, infine, Elisa Cappelli con In Svezia. Impressioni di viaggio. Libro per la gioventü ${ }^{6}$ (1902).

L'austriaca Pfeiffer, la belga Carla Serena, l'inglese Alec-Tweedie e l'italiana Cappelli si ritagliano delle nuove dimensioni all'insegna di una diversa autoconsapevolezza e conoscenza del mondo, eppure riportano nelle loro scritture un continuo tentativo di riduzione di questo atteggiamento ritenuto socialmente inopportuno o trasgressivo. Nonostante i viaggi delle inglesi siano famosi, frequenti e più documentati ${ }^{7}$ rispetto, ad esempio, alle esperienze delle italiane, è indubbio che il viaggio non necessariamente scortato, quasi impensabile nei periodi antecedenti a quello in esame, sia compiuto da donne con una certa personalità, curiosità, formazione e classe sociale, ma anche incoscienza e ostinazione. Tale reputazione si accompagna all'analisi scrutinante dello sguardo femminile sulla vita locale, le culture e le tradizioni di luoghi poco noti, scaturendo poi in pagine diaristiche e nozionistiche di notevole interesse.

Questi resoconti della seconda metà e tardo Ottocento diventano, quindi, dei raccordi tra vissuti e interpretazioni del reale, pur non pervenendo a uniformità stilistiche. Nel presente saggio si vuole indagare il rapporto formale nonché ideologico tra di essi, esplorando le diverse soluzioni a cui le viaggiatrici giungono, tenuto conto di tutti i limiti a cui esse erano sottoposte. Con l'obiettivo di contestualizzare i resoconti odeporici, si partirà dal rispondere a dei semplici quesiti mirati ad analizzare i motivi che le spingono a viaggiare, evidenziandone le modalità (con chi e come) e, successivamente, a scrivere esplorando la presenza dell'io-narrante. Per comprendere le immagini e i temi, si useranno come chiave esegetica le tre categorie proposte da Pratt (1992) nella retorica della scoperta: l'estetizzazione del paesaggio, la ricerca di densità di significato e la padronanza dello scarto tra realtà e aspettative. ${ }^{8}$

\section{Il femminile tra viaggio e società}

Il dibattito sul ruolo della donna in Europa a metà Ottocento è di certo complesso, tanto che tracciarne un quadro esaustivo risulta particolarmente complicato poiché la condizione femminile è strettamente radicata nel tessuto socio-culturale del paese di provenienza. ${ }^{9}$ Altrettanto tortuoso e intricato è il percorso della letteratura di viaggio

Heckenast, 1846 - è disponibile al seguente link http://baekur.is/bok/000311077/2/247/ Reise_nach_dem/?iabr=on\&ui=embed\#page/Saurbla\%C3\%B0++(4+/+276)/mode/2up (20 aprile 2020).

${ }^{4} \mathrm{C}$. Serena, Mon voyage: souvenirs personnels. De la Baltique à la Mer Caspienne, Paris, Maurice Dreyfous, 1881, https://gallica.bnf.fr/ark:/12148/bpt6k28629p/f1.image.textelmage (29 marzo 2020).

${ }^{5}$ E.B. Alec-Tweedie, A Girl's Ride in Iceland, London, Horace Cox, Windsor House, Bream's Buildings, E.C. 1894. Il testo è disponibile nell'ambito del Progetto Gutenberg (https://gutenberg.unblockit.win/ ebooks/26006 12 maggio 2020) in formato mobi. Per convenienza, tale formato è stato convertito, quindi le citazioni faranno riferimento alla versione pdf dell'ebook [\#26006 - 8 luglio 2008].

${ }^{6}$ E. Cappelli, In Svezia. Impressioni di viaggio. Libro per la gioventù, Firenze, Bemporad e Figlio, 1902, p. 1.

${ }^{7} \mathrm{Nel}$ XVIII secolo, tra le illustri viaggiatrici si annoverano: le britanniche Lady Mary Wortley Montagu (16891762), Mary Wollstonecraft (1759-1797) e Ann Radcliffe (1764-1823), nonché l'irlandese Lady Mount Cashell (1773-1835). Nel XIX secolo, si aggiungono, ad esempio, Maria Graham (1785-1842), Elizabeth Strutt (1805-1863), Isabella Bird (1831-1904) e Mary Kingsley (1862-1900). In aggiunta, cfr. Morris \& O'Connor, The Virago Book, cit.

8 'Analyzing Victorian discovery rhetoric, I have found it useful to identify three conventional means which create qualitative and quantitative value for the explorer's achievement. [...] First, and most obvious, the landscape is aestheticized. [...] Second, density of meaning in the passage is sought. [...] [t]he relation of mastery predicated between the seer and the seen' (M.L. Pratt, Imperial Eyes. Travel Writing and Transculturation, London \& New York, Routledge, 1992, pp. 204-205). Si procederà quindi con il metodo del campionamento intenzionale.

9 È innegabile che fenomeni e periodi di varia natura, quali, a esempio, l'llluminismo, la Rivoluzione francese, la proto-industrializzazione sul continente, ma anche le spinte colonialiste abbiano 
ottocentesca ${ }^{10}$ che, in linea temporale, si colloca tra il 'secolo d'oro dei viaggi', ${ }^{11}$ il Settecento, che vede il soffermarsi dello sguardo dei viaggiatori sul passato nel presente - ovvero un viaggio nel tempo tra monumenti e rovine storiche - e la letteratura di viaggio del Novecento, in cui i resoconti puntano anche sulle capacità affabulatorie dei loro autori per fornire una rilettura di luoghi oramai noti anche al vasto pubblico, caldeggiando una prospettiva straniante.

Vi è uno straniamento letterario, teorizzato dai formalisti russi, in particolare da Šklovskij (1893-1984), ${ }^{12}$ fondato sulla concezione di otstranenie, straniamento appunto, che si raggiunge quando i meccanismi interpretativi della percezione della realtà perdono il loro naturale automatismo. A esso, si aggiunge lo straniamento del teatro epico, verfremdungseffekt, postulato da Brecht, ${ }^{13}$ che mira a stimolare un atteggiamento analitico rispetto ai fatti rappresentati sulla scena con tecniche di distanziamento emotivo che offrono allo spettatore l'occasione di sviluppare un pensiero critico. Tuttavia, si ritiene fondamentale esplorare anche uno spaesamento di derivazione percettiva concreta, che, riecheggiando la matrice brechtiana dell'analisi critica, si innesca dalla profonda interconnessione tra spazio e individuo nella loro influenza reciproca. Seppur più fisico e spaziale, esso conduce a forme di straniamento altrettanto significative per cui l'esplorazione effettiva dello spazio e la lente interpretativa socio-culturale del viaggiante portano a esiti dai variegati toni e registri narrativi: 'L'essere "straniero" è già, quasi etimologicamente, una manifestazione di "straniamento", poiché in un paese straniero si vede tutto per la prima volta, e la percezione è dunque più intensa'. ${ }^{14}$

Spazio e tempo vengono filtrati dalla percezione del viaggiat-ore/-rice; di fatto

[l]'esperienza non risiede più nel lungo sviluppo, nel faticoso cammino di formazione, ma nel dettaglio, nell'apparentemente insignificante. $\mathrm{E}$, in fondo, l'esperienza della fugace apparizione in un oggetto di un significato che va al di là di quello quotidiano, che Joyce chiamava Epiphany, non sembra molto lontana da questa ricerca. Tutta la grande letteratura del Novecento, da Proust a Kafka, da Musil a Joyce appunto, riletta in questo senso, appare come una ricerca nell'apparente insignificante, di una autenticità che si rivela nell'oggetto, 0 meglio, nella percezione non automatica, 'straniata', 'epifanica' dell'oggetto. ${ }^{15}$

Le viaggiatrici in esame nel presente saggio giungono da realtà eterogenee. Tra aprile e ottobre 1845, dopo la dovuta preparazione linguistica e scientifica, Ida Pfeiffer $(1797-1858),{ }^{16}$ nata in una famiglia benestante di commercianti viennesi e famosa per

profondamente modificato il ruolo delle donne nella società d'appartenenza; società europee che hanno vissuto gli eventi precedentemente menzionati con modalità e conseguenze dissimili (Cfr. J. Hurl-Eamon, Women's role in Eighteenth Century Europe, Santa Barbara, Greenwood, 2010).

${ }^{10}$ Come nota Rossi: 'Tornando all'Ottocento, la rarità di nomi femminili nelle file delle scienze territoriali ancor più che nelle altre scienze, va appunto ricondotta al carattere marcatamente strategico dei saperi geografici e alla carta come strumento di simbolo del potere piuttosto che al disinteresse delle donne per questi studi o alla presunta incapacità di misurarsi con le difficoltà e le ostilità della pratica del terreno' (L. Rossi, L'Altra Mappa. Esploratrici, Viaggiatrici, Geografe, Reggio Emilia, Diabasis, 2005, p. 28).

${ }^{11} \mathrm{Cfr}$. A. Brilli, Quando viaggiare era un'arte. Il romanzo del Grand Tour, Bologna, Il Mulino, 2017.

12 Cfr. V. Šklovskij, L'arte come artificio, Milano, Garzanti, 1917. Lo straniamento, come artificio sperimentale, ha diverse finalità e si attua sia attraverso interventi sul piano linguistico sia di focalizzazione.

13 Mutuando dal teatro, distacco e distanza diventano le chiavi di lettura dell'esperienza per poter giungere alla conoscenza (Cfr. B. Brecht, Scritti teatrali I, Torino, Einaudi, 1975).

${ }_{14}$ L. Antoccia, Il viaggio nel cinema di Wim Wenders, Bari, Edizioni Dedalo, 1994, p. 35.

15 Ivi, p. 35.

${ }^{16}$ Nata Ida Laura Reyer a Vienna, in Austria, morì nella città austriaca per una malattia che aveva contratto durante il suo ultimo viaggio in Madagascar. Il matrimonio combinato con Mark Anton Pfeiffer, un avvocato di Lemberg (Lviv, Ucraina), 24 anni più grande, durò, anche se ufficiosamente, una decina d'anni. Il primo viaggio, nel 1842, avvenne, inizialmente, sotto il falso pretesto di una visita ad amici a Costantinopoli e, 
essere stata la prima donna europea ad aver esplorato l'interno dell'isola del Borneo, si dirige nel nord Europa, che sembra però deludere le sue aspettative giacché nelle campagne visitate non vi trova l'idillio sperato. Pfeiffer, collezionista di oggetti naturali ed etnografa, tende le rigide maglie della società di metà Ottocento sfruttando l'età, 45 anni al momento del suo primo viaggio, visto che le donne mature erano meno soggette al controllo maschile. ${ }^{17}$ Da quest'esperienza deriva il volume in esame, Visit to Iceland and Scandinavia North, la cui traduzione in inglese è corredata da numerose note esplicative, otto litografie a colori, un saggio sulla poesia islandese, una traduzione della poesia islandese La profezia del veggente, ${ }^{18} \mathrm{e}$ un breve riassunto della storia islandese. Nella prefazione stilata dall'autrice si forniscono le linee essenziali di un quadro composito: di una società non avvezza, come anzidetto, al viaggio di una donna in solitaria, di una viaggiatrice consapevole dei freni esterni imposti, di un'autrice che apre la sua stesura servendo la 'dovuta' giustificazione ai lettori:

'Another journey - a journey, moreover, in regions which everyone would rather avoid than seek. This woman only undertakes these journeys to attract attention'.

'The first journey, for a woman ALONE, was certainly rather a bold proceeding. Yet in that instance she might still have been excused. Religious motives may perhaps have actuated her; and when this is the case, people often go through incredible things. At present, however, we can see no just reason which could excuse an undertaking of this description'.

Thus, and perhaps more harshly still, will the majority judge me. And yet they will do me a grievous wrong. I am surely simple and harmless enough, and should have fancied anything in the world rather than that it would ever be my fate to draw upon myself in any degree the notice of the public. I will merely indicate, as briefly as may be, my character and circumstances, and then I have no doubt my conduct will lose its appearance of eccentricity, and seem perfectly natural.

[...] And now, dear reader, I would beg thee not to be angry with me for speaking so much of myself; it is only because this love of travelling does not, according to established notions, seem proper for one of my sex, that I have allowed my feelings to speak in my defence.

Judge me, therefore, not too harshly; but rather grant me the enjoyment of a pleasure which hurts no one, while it makes me happy. ${ }^{19}$

in un secondo momento, sotto le false spoglie di un pellegrinaggio religioso. L'intenzione della casalinga viennese, divenuta una grande e affermata viaggiatrice, era quello di uscire dalle mura domestiche per esplorare il mondo in modo indipendente. Sulla vita e i viaggi di Ida Pfeiffer si vedano: D. Jedamski, Images, Self-Images and the Perception of the Other: Women Travellers in the Malay Archipelago, Hull (UK), University of Hull, Center for South-East Asian Studies, 1995; Agosin \& J.H. Levison, Magical Sites: Women Travels in 19th C. Latin America, Buffalo (NY), White Pine Press, 2010, pp. 191-206; J. Michaels, 'Ida Pfeiffer's Travels in the Dutch East Indies and Magadascar', in: Austrian Studies, 20 (2012), pp. 6074; J. Michaels, 'An Unsual Traveler: Ida Pfeiffer's Visit to the Holy Land in 1842', in: Quest. Issues in Contemporary Jewish History. Journal of Fondazione CDEC, 6 (december 2013), http://www.questcdecjournal.it/focus.php?id=340 (21 maggio 2020); J. Van Wyhe, Wanderlust. The amazing Ida Pfeiffer. The First Female Tourist, Singapore, NUS Press, 2019.

17 All'epoca, oltre alla già citata Lady Montagu, altre donne, quali Isabella Frances Romer (1798-1852) e Lady Hester Stanhope (1776-1839), viaggiavano con i loro mariti o chaperone di sesso maschile oppure in compagnia della madre come Helen Emily Lowe (-1882), mentre Pfeiffer non solo non avrebbe trovato nel marito un compagno di viaggio - si ricorda che il fallimento del matrimonio e l'età avanzata erano aspetti critici - ma si ritrova spesso in situazioni di viaggio pessime. Pfeiffer affronta il tema dell'età con vena ironica - 'All who are accustomed to the sea will testify that I have adhered strictly to the truth. But to return to the sloop. Its age emulated mine, she being a relic of the last century' (Pfeiffer, Visit to Iceland, cit., p. 128).

18 'Völuspâ' è il primo nonché il più famoso poema gnomico dell'Edda Poetica o Canzoniere eddico, una raccolta di poemi in norreno in cui la veggente ( $v$ glva), interpellata da Odino, evoca la creazione, la distruzione e la rinascita dell'universo (Cfr. M. Polia, La voluspà. I detti di Colei che vede, Rimini, Il Cerchio, 1983).

19 Pfeiffer, Visit to Iceland, cit., pp. 35-36. 
Una simile premessa pre-partenza giunge da Mrs Alec-Tweedie, ovvero Ethel Brilliana Tweedie (1862-1940), ${ }^{20}$ che, invece, a 24 anni, deve ricorrere al sostegno del fratello ${ }^{21}$ per poter affrontare la visita dell'Islanda nel 1886. Con conoscenze di rilievo nel campo dell'esplorazione, la viaggiatrice inglese, dopo una stagione londinese, ${ }^{22}$ attende con trepidazione il viaggio verso 'Ultima Thule'. ${ }^{23}$ Oggi è forse conosciuta maggiormente per i suoi resoconti di viaggio, ma fu anche fotografa, pittrice e filantropa - esempio di come la classe fosse essenziale in quanto la scrittrice indugia talora su aspetti triviali, quali la scomodità dei trasporti, gli acciacchi dovuti al viaggio, talora su attività sportive, come l'andare a cavallo e lo sci, narrate in modo tale da poter spostare l'attenzione dalla violazione delle tipiche convenzioni vittoriane. Il volume di Tweedie è composto da XII capitoli con varie sezioni suddivise per argomento e un'appendice tematica sui geyser redatta dal padre, Dr George Harley. Mondana, talvolta estrosa, l'autrice spazia dai mezzi di trasporto alla moda, dal paesaggio ai costumi riportando con chiarezza espositiva, precisione pseudo-scientifica ${ }^{24}$ e schiettezza di giudizio le sue impressioni sull'Islanda.

Spesso paragonata a Pfeiffer, Carla Serena (nom de plume di Caroline Hartog Morgensthein, 1820-1884), belga di nascita e italiana d'adozione, ${ }^{25}$ comincia a viaggiare da sola sulla cinquantina; nel 1874 è ospite a Stoccolma di Re Oscar II (18291907) e, successivamente, si reca sul Mar Caspio e sul Mar Nero dove per due anni studia la popolazione locale. In linea con le prime due autrici, anche la scrittrice belgoitaliana conferma la peculiarità del suo viaggio in solitaria fornendo, tuttavia, una nota sulle sue modalità, tipiche del periodo in considerazione, basato sulle lettere di

\footnotetext{
20 L'estesa bibliografia dell'autrice, dal 1889 al 1936, include diversi articoli e volumi (A Girl's Ride in Iceland, 1889, A Winter Jaunt to Norway, 1894, e Through Finland in Carts, 1897) che offrono un contributo sostanziale nella definizione della cultura finno-scandinava e ridefinizione dei confini europei per il pubblico di lingua inglese. Cfr. A. Ryall, 'Europe's Northern Periphery and the Future of Women in the Travel Narratives of Ethel Tweedie', in: NORA - Nordic Journal of Feminist and Gender Research, 17, 4 (2009), pp. 273-288.

21 Il gruppo è composto da cinque viaggiatori tra cui, oltre a Tweedie, si annovera una sola donna, Miss T., il futuro marito e il fratello, cosi descritto: "First, my brother, whom, for convenience sake in these pages, I will call by his Christian name, "Vaughan", and whom I looked upon as the head of the expedition, as, without his protection, I should never have been allowed to undertake the trip. He was a medical student in Edinburgh (since fully qualified), and well suited to the enterprise, being of a scientific turn of mind, as well as practical and energetic, -a first-rate rider, an oarsman, and a good sailor, whilst he had spent his vacations for some years in travelling' (Alec-Tweedie, A Girl's Ride, cit., p. 17).

22 Tweedie aveva sperato di poter incontrare il famoso esploratore norvegese, Fridtjof Nansen (18611930), proprio durante la stagione.

${ }^{23}$ Cosi come per R.F. Burton (1821-1980), l'Ultima Thula era rappresentata da 'a holiday in the emptiness for the Victorians, the northern lands a playground for summer trippers in crinolines and smart collars' (J. Kavenna, The Ice Museum: In Search of the Lost Land of Thule, London, Penguin, 2005, p. 55).

${ }^{24} \mathrm{Nel}$ quartetto in esame, Tweedie è l'autrice più ligia nel fornire indicazioni geografiche (es. 'Iceland lies between N. Lat. 63, 23, 30, and 66, 32; and W. Long. 13, 32, 14, and 24, 34, 14; is 280 miles in length, and 180 to 200 miles in breadth', Tweedie, $A$ girl's ride, cit. p. 29), di livello pratico sulle spese (es. 'our tickets were at once procured; not expensive by any means, being only $£ 8$ each person to Iceland and back, including the trip round the Island; our food being charged at the rate of $6 \mathrm{~s}$. 6d. per day extra', ivi, p. 22), e sulle qualità, condizioni e difficoltà dei trasporti.

${ }_{25}$ Caroline Hartog Morgensthein nasce ad Anversa ma nel 1848 si trova a Venezia quando sposa Leone Serena, proscritto dopo il crollo della Repubblica di San Marco. Nel 1858 la famiglia è a Londra dove giunge dopo Francia e Belgio. Assunta come corrispondente per un giornale di Anversa, Carla Serena, così si firmerà sempre, comincia il suo viaggiare da sola per il mondo (Cfr. M. Scriboni, 'Il Viaggio Al Femminile Nell'Ottocento: La Principessa Di Belgioioso, Amalia Nizzoli e Carla Serena', in: Annali D'Italianistica, 14 (1996), pp. 304-325). All'età di 50 anni parte per un lungo viaggio solitario di sei anni nell'Impero Ottomano (1874-75), nell'Impero Russo $(1874,1875-77$, 1878-79, 1881) e nell'Impero Persiano (1877-78). Sull'autrice, si vedano, inoltre, il volume di D. Pizzagalli, Il viaggio del destino: Carla Serena da Venezia al Caucaso, Milano, Rizzoli, 2006 e il saggio di F. Ardillier-Carras, 'Carla Serena. La prima donna in viaggio solitario nel Caucaso’, in: F. Frediani, R. Ricorda \& L. Rossi, Spazi, Segni, Parole. Percorsi di Viaggiatrici Italiane, Milano, Franco Angeli, 2012, pp. 51-64.
} 
raccomandazione: 'Je partis seule. Pour une femme un voyage lointain étant toujours hasardeux, je m'assurai moralement. Munie de lettres amicales, je me mis, en outre, sous l'égide officielle de plusieurs puissances par des recommandations d'ambassadeurs, ministres, etc. '.26 Il volume è diviso in nove sezioni, di cui la prima d'interesse per il presente saggio ('En Scandinavie et en Russie'), precedute da quattro pagine in cui l'autrice spiega come si sia rivolta alla scrittura, e seguite da un epilogo. ${ }^{27}$ A cavallo tra i due secoli, in Italia, nazione dalle forti dicotomie - Nord/Sud, Stato/Chiesa, città/campagna, scienza-tecnologia/religione, società patriarcale/ emancipazione femminile ${ }^{28}$ - il viaggio per le giovani donne veniva scoraggiato; le viaggiatrici tra Sette e Ottocento ${ }^{29}$ che contribuiscono alla diffusione della pratica sono di fatto poche e si dividono in coloro che si spostano per lavoro, le artiste professioniste (attrici, pittrici), oppure donne a seguito delle famiglie, come Amalia Nizzoli (18061845) in Egitto o Cristina Trivulzio di Belgiojoso (1808-1871), o coloro che praticano un tour culturale, come Isabella Teotochi Albrizzi (1760-1836). ${ }^{30}$ Certa è la classe sociale a cui queste donne appartengono: dalle dame salonnières ${ }^{31}$ alle borghesi altolocate. $\grave{E}$ in questo contesto che Elisa Cappelli, insegnante ed educatrice fiorentina, s'inserisce con il suo resoconto - corredato da 28 fototipie, pubblicato nel 1902 dopo che l'autrice si era recata in Scandinavia nel $1898^{32}$ - fedele al suo ruolo educativo sia negli intenti, poiché i destinatari sono i discenti, sia nella forma, nello sforzo di perpetrare una forzata obiettività. Cappelli viaggia in compagnia di Ebba Atterbom, ex-allieva e amica svedese, a cui il volume è dedicato. L'io errante-narrante crea sin dalle prime righe una comunanza con il femminile 'straniero', tanto da descrivere la sua compagna di viaggio come 'più italiana che svedese, sia per la consuetudine dell'aver abitato molto tempo a Firenze, come per il sentimento'. ${ }^{33}$

Diversi sono i mezzi di trasporto utilizzati e descritti: dagli omnibus ${ }^{34}$ - presi da Pfeiffer alle cinque del mattino del 10 aprile 1845 - alle imbarcazioni di vario genere, carri, tram di circonvallazione e treni - su cui Cappelli sale per la prima volta -

${ }^{26}$ Serena, Mon voyage, cit., p. 6. Fondamentali saranno i contatti altolocati che la introdurranno in cerchie d'élite, a partire dal Re Svedese, il quale, interessato al suo viaggio, l'accolse con cordialità e pianificò la sua visita in Norvegia, luogo da cui si accomiatò in illustre compagnia: 'Le jour de mon départ, à cinq heures du matin, je le [le chambellan] vis à la gare m'attendant un bouquet en main. Je pris place dans une voiture de première. M'y voyant seule, il chercha quelqu'un pour me mettre sous sa protection apercevant le poète norvégien Ibsen, installé avec sa famille dans un wagon de seconde, il les pria de passer dans mon compartiment, [...]' (Serena, Mon voyage, cit., p. 9). Con l'approvazione del Re, questo soggiorno tra Svezia e Norvegia verrà pubblicato con il titolo di Lettres Scandinaves (1874).

27 Carla Serena ribadisce la sua posizione eurocentrica, esplicitata in precedenza nella dicotomia noiEuropei/ loro-Turchi: 'De l'Europe j'étais partie. En Europe je suis revenue, heureuse d'avoir réalisé mon but. A l'Europe afflue tout ce que l'esprit humain crée, invente, exécute. A l'Europe, souveraine du monde de la-pensée, sont présentes les créations des intelligences supérieures et tout modeste labeur. L'Europe, centre suprême de la civilisation ressemble à la vaste mer où arrive aussi bien que le tribut du fleuve grandiose le plus mince filet d'eau. Tout y trouve place. A l'Europe donc je présente aussi mon faible travail' (Serena, Mon voyage, cit., p. 358).

${ }^{28} \mathrm{Cfr}$. C. Gori, 'Oltre domani: futuro, progresso e divino nell'emancipazionismo italiano tra Otto e Novecento', in: Storia delle donne, 1 (2005), pp. 239-255.

${ }^{29}$ Cfr. Frediani, Ricorda \& Rossi, Spazi, Segni, Parole, cit.

${ }^{30}$ R. Ricorda, Viaggiatrici italiane tra Settecento e Ottocento. Dall'Adriatico all'altrove, Bari, Palomar, 2011, p. 17.

31 Ivi, p. 18.

32 La prima annotazione risale all'8 giugno 1889, partenza da Firenze e direzione Monaco; attraversando la Germania e la Danimarca, raggiunge la Svezia. L'ultima annotazione è del 29 luglio di ritorno a Firenze. Tale precisione temporale cronachista è peculiare del resoconto cappelliano, presente con lassi temporali importanti in Pfeiffer e pressoché assente in Tweedie e Serena, nei cui volumi si prediligono i sottotitoli. ${ }^{33}$ Cappelli, In Svezia, cit., p. 1.

34 'Our omnibuses are so small and narrow, that one would suppose they were built for the exclusive accommodation of consumptive subjects, and not for healthy, and in some cases portly individuals, whose bulk is further increased by a goodly assemblage of cloaks, furs, and overcoats' (Pfeiffer, Visit to Iceland, cit., p. 7). 
importanti in quanto ricreano forme di socialità temporanee, permettendo alle viaggiatrici di venire a contatto con l'Altro e cambiano la finestra d'osservazione, introducendo inoltre il concetto di velocità nel movimento tanto fisico quanto narrativo. Tali annotazioni pratiche (i mezzi disponibili per alcune tratte, i costi dei biglietti, le tempistiche e le modalità) diventano dunque utili nella rappresentazione della pratica ma gettano anche le basi per confronti socio-culturali:

[...]the distances are long, the roads bad, and large rivers, moorlands, and swamps must frequently be passed; so every one rides, both men, women, and children. The use of carriages is as totally unknown throughout the island as in Syria. ${ }^{35}$

La connessione spaziale e culturale proposta da Pfeiffer tra Islanda e Siria comporta uno scarto non solo geografico ma immaginativo: il lettore, infatti, deve proiettarsi all'improvviso in un'area ben diversa e crearsi una nuova immagine mentale. Tale artificio - ovvero l'imporre un'associazione che porta colui che legge a sentirsi temporaneamente spaesato - viene, inoltre, stimolato dall'intertestualità e la referenzialità, ${ }^{36}$ caratteristiche essenziali nella produzione odeporica del periodo, che evidenziano altresì l'estrazione socio-economica delle viaggiatrici.

Nei resoconti di esperienze di viaggio in forma di journal intime, una forma in primis intimistica e poi divulgativa, l'lo viaggiante corrisponde con l'io narrante; il narratore diventa dunque portatore di significato e significante. Nonostante l'uso della prima persona, va ricordato che a giocare un ruolo importante nella stesura di un diario di viaggio, ma soprattutto nella sua rielaborazione per la pubblicazione, è il 'lirismo del viandante', ${ }^{37}$ ovvero una libertà poetica di cui il viaggiatore-scrittore può avvalersi. L'lo narrante è in costante ed esplicito dialogo con il suo lettore in Pfeiffer - 'My readers must bear in mind, however, where I came from, and how long I had been imprisoned in a vessel in which I scarcely had space to move',$-{ }^{38}$ e anche in Tweedie, ${ }^{39}$ benché in forma meno preponderante - 'It may be well here to introduce our party individually to my readers'. ${ }^{40}$ Questo dialogare con il proprio lettore è assente, invece, in Cappelli, la quale oscilla tra prima persona singolare e plurale e, in caso di necessità, predilige le interiezioni e le domande retoriche, come in Carla Serena, che ricorre piuttosto ai dialoghi - inserendo una plurivocità e uno sdoppiamento enunciativo.

\section{Il Nord di Pfeiffer, Serena, Tweedie e Cappelli a confronto}

Il nord Europa diventa fonte di interesse pur restando meta inconsueta. Svariati erano i riferimenti letterari ${ }^{41}$ per le viaggiatrici inglesi - dalla prosa alla narrativa di viaggio,

\footnotetext{
35 Pfeiffer, Visit to Iceland, cit., p. 49. Lo stesso accade in altre circostanze, ad esempio, nel visitare Stoccolma, Pfeiffer paragona due palazzi reali, svedese e napoletano: 'It would fill a volume were I to enumerate and describe the treasures, curiosities, and beauties of its construction, or its interior arrangement; I can only say that I never saw any thing to equal it, except the royal palace of Naples' (Pfeiffer, Visit to Iceland, cit., p. 162).

36 Per intertestualità e referenzialità s'intende 'l'esito organico della commistione degli appunti presi in loco e di quella che si potrebbe definire la biblioteca universale del viaggiatore' (E. Patat, 'La visionarietà della parola in Istanbul. Il doppio viaggio di Adele Cambria', in: M. Marino \& G. Spani (a cura di), Donne del Mediterraneo. Saggi interdisciplinari, Firenze, Società Editrice Fiorentina, 2016, pp. 76-83; 76).

${ }^{37}$ Ricorda, Viaggiatrici italiane, cit., p. 12.

38 Pfeiffer, Visit to Iceland, cit., pp. 6, 20, 36, 44, 59, 91, 105, 113, 128, 130, 133, 139, 152, 161, 188, 190, 205.

${ }^{39}$ Alec-Tweedie, A Girl's Ride, cit., pp. 17, 36, 40, 47, 78, 87, 90.

40 Ivi, p.17.

${ }^{41}$ Alla metà del secolo, risale Feats on the Fjord: A Tale of Norway (1841) di Harriet Martineau (18021876), mentre verso la fine del secolo si trova A Hardy Norseman (1889) di Edna Lyall (1857-1903) (P. Fjågesund \& R.A. Syme, The Northern Utopia: British Perceptions of Norway in the Nineteenth Century, Amsterdam-New York, Rodopi, 2003, pp. 73-75).
} 
alle guide turistiche - richiesti soprattutto da molti turisti che affollavano i fiordi ${ }^{42}$ poiché divenuti di moda per l'attività fisica all'aria aperta. Questa crescente attenzione non coinvolge "tutto" il nord; nell'incipit Alec-Tweedie scrive:

When I ventilated the idea outside my private 'den,' wherein it first arose, it was treated as far too wild a scheme for serious consideration -for 'Iceland,' to Londoners, seems much the same in point of compass as the moon! And there really is some similarity in the volcanic surface of both. Here, however, the similarity ends, for while the luminary is indeed inaccessible, the island can easily be reached without any very insurmountable difficulty. ${ }^{43}$

Certo, non si parla della zona dei fiordi - nel brano citato, infatti, la meta è l'Islanda -, tuttavia il paragone alla superficie lunare è d'indubbia forza evocativa, determinando la sublimazione di un luogo remoto così distante esteticamente dalla quotidianità. Si noti, inoltre, la poetica del contrasto, ovvero la tendenza a presentare un luogo, un paesaggio o un oggetto, descrivendone alcune caratteristiche per poi smentirle 0 limitarne la validità nella continua logica oppositiva tra conosciuto e sconosciuto. In questa realtà lunare, le donne sono presenza ben rara: 'Ladies are such rare visitors in Iceland, that little or no preparation is made for their comfort', ${ }^{44}$ annota AlecTweedie. E già in quest'ampia area geografica, in un 'Nord' in senso lato, si evidenziano le prime gerarchie 'turistiche', luoghi che sono più frequentati di altri; dato rilevante in quanto lo spaesamento del soggetto viaggiante è direttamente proporzionale alla popolarità dei luoghi visitati.

\section{L'estetica del paesaggio}

Come sottolinea Pratt (1992), nel resoconto il piacere estetico della vista diventa il valore e il significato stesso del viaggio. ${ }^{45}$ La conformità dei canoni estetici emerge nelle descrizioni di diapositive di quotidianità:

Quivi [Monaco di Baviera], come ordinariamente nei paesi del Nord, sogliono esporre i fiori in vasetti eleganti di tutte le fogge e di tutte le grandezze, che sembrano messi lì come per ornamento di un salotto. E sotto i fiori scintillavano anche i vasi incisi e dipinti, essendo i lavori sul vetro una delle industrie più sviluppate in questa città. ${ }^{46}$

Cappelli qui offusca i confini spaziali - mescolando esterno e interno - così come le dimensioni in un gusto prettamente femminile del decoro e nel contempo accenna allo sviluppo industriale tipico della zona. L'eleganza, la brillantezza e la ricercatezza dei vasi di vetro e la policromia dei fiori in essi riposti vengono indicati come tratti 'ordinari' dei paesi che si appresta a visitare. È compito del 'pittore verbale' rendere 'estremamente significativo ciò che è, soprattutto dal punto di vista narrativo, praticamente un non-evento' ${ }^{47}$ Soffermarsi sugli oggetti d'ordine comune sembra smorzare la potenziale percezione di spaesamento dell'io viaggiante canalizzandolo nuovamente nella sfera domestica, spazio normativo per eccellenza. Il resoconto cappelliano si fonda sulla ripetizione dei dettagli: minuziosi sono i particolari e ristretti i lassi temporali tra le azioni; la tendenza è di guidare il lettore nell'esperienza vissuta, in cui l'indugiare su elementi noti - siano essi architettonici, naturali, comuni, spesso banali - ricrea un senso di familiarità pur riportando lo stupore o l'idiosincrasia verso

\footnotetext{
42 Ivi, p. 74.

${ }^{43}$ Alec-Tweedie, A Girl's Ride, cit., p. 17.

44 Ibidem.

45 'It is important to note that within the text's own terms the aesthetic pleasure of the sight singlehandedly constitutes the value and significance of the journey' (Pratt, Imperial Eyes, cit., p. 204).

${ }^{46}$ Cappelli, In Svezia, cit., p. 6.

47 Pratt, Imperial Eyes, cit., p. 202.
} 
episodi in cui l'io narrante è consapevole del suo essere straniero. In contrasto, gli altri resoconti, in alcuni brani veri e propri manuali, forniscono nozioni più storiche, letterarie, scientifiche e tecniche.

Dal sud al nord della Germania, il paesaggio sembra mutare; Pfeiffer scrive:

The first fourteen miles are very agreeable; gardens, fields, and meadows, pine forests in the plain and on the hills, and between these, villages, farms, country houses, and solitary chapels, combine to form a very pretty landscape. But the scene soon changes [...]. From here to Leipzig we travel through a wearisome monotonous plain, enlivened at long intervals by villages and scattered farms. There is nothing to see but a great tunnel, and the river Pleisse - the latter, or rather the Elster, is rendered famous by the death of Prince Poniatowski. ${ }^{48}$

Natura e storia si mescolano in pagine odeporiche dai passaggi spaziali ed emozionali anche qui repentini. Per Pfeiffer, la commerciale Leipzig è affollata, 'disfigured with announcements, in all sizes and shapes, covering its walls';49 la grandeur della città viene sminuita nonostante le imponenti costruzioni architettoniche.

Parte integrante dei paesaggi nordici sono i suoni: mentre le pagine di Cappelli risuonano di musica - le bande, ad esempio, usate a pretesto per le descrizioni fisiche dei loro componenti - Pfeiffer, più critica, sembra ridurre la presenza musicale sovrapponendovi caratteristiche prevalentemente negative dei luoghi visitati, e Tweedie ne fa appena un accenno a Reykir, nel nord dell'isola - 'Curiously enough, there is little or no native music, however'.$^{50}$ Diversa è la casistica per Serena, la quale riporta musica in occasione di eventi importanti in compagnia di altrettanto illustri personalità. Al di fuori dai centri abitati, tuttavia, i suoni si affievoliscono lasciando spazio al solo sguardo.

La natura del Nord viene descritta soprattutto per i suoi colori predominanti - le gradazioni dal verde al blu; si prenda come esempio una delle innumerevoli descrizioni elaborate da Alec-Tweedie:

So calm and beautiful was the scene, that all our party agreed it was worth a few days' discomfort in order to revel in the beauty of this bold Icelandic approach. The water was perfectly green, and as clear as possible, revealing innumerable yellow jelly-fish disporting themselves. We did not, however, see any of the sharks which are so frequently met with in these waters. ${ }^{51}$

In questo passaggio, ma si potrebbe generalizzare e applicare questa tendenza a tutti i resoconti analizzati, la vista, mediante un campo visivo ampio e inclusivo, e l'udito sono predominanti nell'osservazione dell'ambiente; nel brano sopracitato la presenza umana non irrompe, così come è assente una fauna potenzialmente inquietante. Se in Cappelli, Pfeiffer e Alec-Tweedie natura e architettura, esterno e interno, si controbilanciano, in Serena, più mondana e socialmente inserita, si ripresentano, in maggioranza e nella sezione in esame, ambienti vissuti e chiusi.

Interessante è l'attenzione che Cappelli rivolge ai fiori; menzionati en passant da Alec-Tweedie - 'Although there are no butterflies, and but few insects, flowers abound ${ }^{52}$ - e osservati con curiosità scientifica da Pfeiffer, sono caricati di significati plurimi, fino a farle affermare commentando l'uso di donarli o esporli: 'Gli Svedesi sentono la religione delle cose poetiche e gentili'. ${ }^{53} \mathrm{Ai}$ fiori affida anche il compito di

\footnotetext{
48 Pfeiffer, Visit to Iceland, cit., p. 15.

49 Ibidem.

50 Alec-Tweedie, A Girl's Ride, cit., p. 52.

51 Ivi, p. 29.

52 Ivi, p. 38.

${ }^{53}$ Cappelli, In Svezia, cit., p. 44.
} 
una fisicità stereotipica: 'Nell'uscir da lì incontrammo dei bimbi timidi e rispettosi che s'inchinavano al nostro passaggio, con un inchino che pareva fatto a molla. Avevano gli occhi azzurri come il cielo e come i fiori che ornano i loro prati'. ${ }^{54}$

Nell'eventualità in cui il paesaggio perda il suo fascino, Cappelli viaggiatrice ridimensiona l'elemento negativo:

lo mi sgomentavo ad andare avanti. Il piede affondava fra le pozzanghere di un terreno ineguale e ciottoloso, fiancheggiato da lunghi boschi di abeti e laghi e prati. Il paesaggio pittoresco sotto quell'imperversar di natura diveniva orrido, senza essere meno attraente; ma non c'era tempo né voglia di osservare, tanto ci sentivamo impazienti, ed io per la prima, di arrivare a questa benedetta stazione a cui non giungevamo mai. ${ }^{55}$

Impietose invece l'austriaca e l'inglese che, ritrovandosi in situazioni meno comode, raccontano in toni critici la monotonia, la povertà, le condizioni di vita dei luoghi visitati.

\section{La ricerca di significato}

La densità di significato si esplica in rappresentazioni estremamente ricche di sostanza materiale e semantica. ${ }^{56}$ L'uso di modificatori aggettivali e l'introduzione di oggetti o materiali aggiuntivi nel discorso narrativo contribuiscono all'alterazione di tratti significativi di base caricandoli di sfumature di ordine soggettivo e migliorativo.

Questa tendenza è manifesta soprattutto nelle pagine italiane. Riprendendo l'atmosfera quasi lunare suggerita da Tweedie in merito all'Islanda, il paesaggio di un mondo 'altro' sembra emergere in Cappelli: 'Se non fosse il bisogno di dormire, in Svezia, specialmente in campagna, metterebbe conto passar le notti all'aperto, tanto è dolce sognare e fantasticare sotto quel cielo bianco, bianchissimo senza luna e senza stelle'. ${ }^{57}$ Le ripetizioni e l'enfasi cromatica procurata dal superlativo insieme all'implicito chiarore vacuo conducono a una potenzialità d'azione seppur irreale in un ambiente quasi latteo. In una logica espositiva che si fonda sulla sottrazione - del sonno e degli astri - e sulla dialettica vicinanza-lontananza, ci si avvicina a una dimensione onirica, indefinita, che sembra riflettere un'idealità esistenziale: risalta, infatti, nel resoconto cappelliano la tendenza a idealizzare i paesi del Nord, le sue popolazioni, le abitudini e i costumi. Più mondana ma pur sempre idillica la seguente immagine:

Le donne di servizio eran cariche di vettovaglie, e sull'erba folta un vasto prato, all'ombra di alberi secolari, fu imbandita la mensa copiosa e succulenta, inaffiata dal vino del Reno e dall'allegria schietta dei convitati, circa una ventina, fra uomini e signore. ${ }^{58}$

Lo sguardo della viaggiatrice italiana, nella generale positività espressa nei confronti dei luoghi visitati e delle persone incontrate, coglie passaggi in cui elementi naturali e presenza umana si mescolano, facendo emergere scene di armoniosa convivialità. Una socialità contenuta, mai esagerata, e spesso in osmosi con l'ambiente circostante. 'La foresta è silenziosa, il paesaggio è grandioso, solenne, calmo come gli abitanti di queste placide regioni'. 59

Alterazioni osmotiche investono, contestualmente, elementi architettonici e umani: 'Con un treno da bambole, tanto era piccino e breve (una specie dei nostri trams con due o tre carrozze) fummo in un paesello vicino a Ludvika a vedere una

\footnotetext{
54 Ivi, p. 92.

55 Ivi, p. 52.

56 Pratt, Imperial Eyes, cit., p. 203.

57 Cappelli, In Svezia, cit., p. 122.

58 Ivi, p. 20.

59 Ivi, p. 96.
} 
vecchina, la quale abitava una casetta tutta per sé' ${ }^{60}$ Ridimensionato dal circoscritto sguardo d'osservazione, il paesaggio e i suoi oggetti risentono, anche linguisticamente tramite le forme diminutive, di tale riduzione. Affascinata dal treno ma spaesata nel suo uso all'inizio del viaggio, Cappelli crea una percezione diversa dell'oggetto, esprimendo intimità rievocando una dimensione giocosa e fanciullesca prima, quasi fiabesca poi.

\section{Tra aspettative e realtà}

Il movimento nello spazio, uno spostamento puramente geografico, diventa un vero e proprio displacement. La prevalenza dell'lo delle autrici che avviluppano la più arida trattazione saggistica con le proprie emozioni, come si è visto nei confronti del paesaggio e degli individui che gli danno vita, parte da istanze vissute precise per sfociare in interpretazioni talvolta pregiudizievoli della realtà circostante. La visione della realtà Altra che le viaggiatrici percepiscono e riportano poi su carta non solo è, di fatto, modellata dalle loro esperienze pregresse e dall'lo viaggiante-narrante ma, soprattutto, risulta essere fondamentale nella creazione della (tras)formazione identitaria e anche nella diffusione di un modello socio-culturale:

Le dépaysement désigne donc le sentiment du personnage/voyageur qui vit un écart entre la réalité imaginée et le monde réel, entre l'espace mental et l'espace réel; il s'agit d'un écart qui souvent amène d'une part à la détérioration des représentations classiques de lieux auparavant inconnus et de l'autre à la construction d'une nouvelle image de soi-même. ${ }^{61}$

Tutte e quattro le viaggiatrici sono consapevoli della loro posizione esterna 'altra', portatrici di un punto di vista etico (da outsider) ${ }^{62}$ una citazione per tutte spiega la loro posizione: 'I had seen things which never occur in our common life, and had met with people as they are rarely met within their natural state'. ${ }^{63}$ Uno 'stato naturale' loro, diverso, che crea distonie, innanzitutto, di matrice culturale derivanti dalle disuguaglianze nelle abitudini: nel desinare ${ }^{64}$ nel viaggiare,${ }^{65}$ nel fare acquisti, ${ }^{66}$ a esempio, che vergono sul confronto-scontro tra sistemi socio-culturali dissimili,

\footnotetext{
60 Ivi, p. 128.

${ }^{61}$ A. Giro, 'L'influence de Pétrarque dans Le venturier au sommet de Michaël La Chance. Dépaysement et étrangisation dans la représentation du Mont Ventoux', in: Viaggiatori Journal. Circolazioni scambi ed esilio, 3, 2 (marzo 2020), pp. 170-207; 172.

62 In chiave antropologica, si distingue tra un punto di vista etico (dell'outsider), tipico dell'osservatore esterno che è 'altro' rispetto alla società osservata, ed emico (dell'insider), del soggetto parte della società osservata (M. Aime \& D. Papotti, L'altro e l'altrove, Torino, Einaudi, 2012, p. 136).

${ }^{63}$ Pfeiffer, Visit to Iceland, cit., p. 190.

64 'Quel sistema di servirsi e di mangiare, nuovo a noi italiani, mi fecero un effetto singolare' (Cappelli, In Svezia, cit., p. 35), in un teatro a Leipzig in presenza di uno spuntino singolare - 'I could not believe my eyes, and had made up my mind that the sausages were artificially formed out of some kind of confectionery - but alas! my nose came forward but too soon, as a potent witness, to corroborate what I was so unwilling to believe!'(Pfeiffer, Visit to Iceland, cit., p. 16); si abbandona la descrizione dell'oggetto (una salsiccia ritenuta inappropriata per il luogo), cedendo all'olfatto il potere descrittivo; o ancora 'Alas! we had no spoons, and how to eat our cream and apricots was a puzzle. Our guide, whom we had christened 'Johnny,' to his great delight, helped us out of this difficulty' (Alec-Tweedie, A Girl's Ride, cit., p. 77). Non solo emergono le diversità culturali ma anche la predominanza socio-culturale tipica del viaggiatore europeo che riporta la realtà al suo conosciuto - qui assegnando un nuovo nome alla propria guida.

65 'Si mon voyage, entrepris seule, avait été un sujet d'étonnement en Europe, peu s'en fallut qu'en Asie ce fait ne me fît déclarer une héroïne' (Serena, Mon voyage, cit., p. 52). Dal suscitare stupore a diventare un'eroina, l'alterazione è strettamente legata all'impresa e dunque alla dimensione spaziale nonché al soggetto coinvolto. Ingrandita esponenzialmente, l'autrice è consapevole dell'unicità del suo viaggio e della sua posizione.

66 'Much of the sale was carried on by barter, a system of trading not wholly comprehensible to us strangers' (Alec-Tweedie, A Girl's Ride, cit., p. 46).
} 
evidenziando la propensione a istanze dicotomiche - primitività/modernità, arretratezza/emancipazione - e pregiudizievoli. ${ }^{67}$

Vi è inoltre uno scarto legato alla topicità che produce effetti dissimili seppur spesso convergenti, espressi con tecniche diverse:

Mi levai col sole già alto spaventata per il timore che fosse troppo tardi. Invece erano le prime ore della mattina, e bisognava ch'io m'assuefacessi a vedere il sole sorgere prestissimo e tramontare ad ora tarda. ${ }^{68}$

Disorientata dalla presenza del sole sopra l'orizzonte, Cappelli ammette la necessità di abituarsi al fenomeno di tali latitudini. ${ }^{69}$ Pfeiffer ispira, invece, un sentimento più suggestivo chiamando in causa la 'dea della luce', ingaggiando la fantasia del lettore: 'To-day I still rose with the sun; but that will soon be a difficult matter to accomplish; for in the north the goddess of light makes amends in spring and summer for her shortcomings during the winter'. ${ }^{70}$

Nello studio dei diari di viaggio di penne femminili, la ricerca del pensiero, dell'interpretazione e del giudizio che le autrici riportano sulle donne degli altri paesi, una sorta di 'fusione' tra punti di vista etici ed emici, non è un aspetto marginale. Nei quattro resoconti si delineano posizioni diverse. Carla Serena tende a porre attenzione alla condizione delle donne, ritenuta indicatore di civiltà; tuttavia, nella sezione in esame poco emerge sull'argomento. Elisa Cappelli è altresì accurata nell'osservare gli individui, partendo da una considerazione generale: 'Si suol dire che i nordici' ${ }^{71}$ sono freddi; io non potrei asserirlo,72 svelando quindi la sua chiara posizione critica. Ciò nonostante, per quanto concerne le figure femminili che popolano il suo viaggio sembra esserci un'oscillazione significativa di pensiero: al 19 giugno risale una testimonianza di matrice tradizionalista - ovvero 'In questo paese, ove i giorni si prolungano straordinariamente in estate quanto le notti nell'inverno, la donna, dedita alla casa, pone ogni sua cura e pensiero nell'abbellirla e far sì che l'uomo, tornando stanco dal lavoro assiduo della giornata, trovi in essa dolce e riposato asilo,73 - in cui la donna è dominante nella cura della casa e, in linea con le usanze del tempo, al servizio del lavoratore. Le mura domestiche, regno femminile e rifugio maschile, rimarcano i classici ruoli della società patriarcale. Eppure, il 24 giugno, sempre a Leksand, in riva

${ }^{67}$ A esempio, a Reikjavik Pfeiffer nota: 'I was much surprised to find that nearly all the Icelanders can read and write' (Pfeiffer, Visit to Iceland, cit., p. 123).

${ }^{68}$ Cappelli, In Svezia, cit., p. 41 (Gothemburgo, 16 giugno).

69 Il bisogno di 'abituarsi' viene ripreso anche da Pfeiffer: 'At first it appeared strange to me to go to bed in broad daylight; but I soon accustomed myself to it' (Pfeiffer, Visit to Iceland, cit., p. 53).

70 Pfeiffer, Visit to Iceland, cit., p. 24. Contribuisce anche Alec-Tweedie - 'At 10.30 p.m. the sun had not yet set, but was shedding its glorious evening glow over mountains which rose almost perpendicularly from the sea, and whose snowclad peaks caught the rosy hues and golden tints of departing day' (AlecTweedie, A Girl's Ride, cit., pp. 28) - insistendo su forme geometriche e varie tonalità, optando per scelte semantiche dal forte potere evocativo.

${ }^{71}$ A più riprese, Cappelli sottolinea le qualità del popolo svedese - 'Un popolo che ha tali principi - pensavo - non può essere che forte e buono, e tali sono gli Scandinavi, non corrotti dal clima o dalle raffinatezze soverchie' (Cappelli, In Svezia, cit., p. 120) - associando non solo i principi, come quelli qui menzionati che si riferiscono al rispetto che i figli dimostrano ai genitori con saluti, baciamano, e prontezza nel servire, bensi anche le caratteristiche fisionomiche. Tale tendenza descrittiva induce a pensare che Cappelli avesse familiarità con le teorie diffuse del secolo, in particolare di fisiognomica e frenologia, elaborate da Lavater a Lombroso, che mettevano in relazione l'aspetto fisico dell'individuo con i suoi caratteri piscologici e morali (Cfr. R. Perugi, 'Quando le italiane arrivarono al Nord; diari delle prime turiste in Scandinavia', in: Settentrione: rivista di studi italo-finlandesi, 28 (2016), pp. 313-324, 317). Il quadro si amplia poi all'ambiente: 'Infatti gli Svedesi, sì per la loro posizione geografica come per l'indole e per l'indirizzo dato alla loro educazione, sono un popolo talmente disciplinato che rifugge da qualunque dissidio o turbamento' (Cappelli, In Svezia, cit., p. 158).

72 Ivi, p. 47.

73 Ivi, p. 56. 
al lago nel vedere le barche, afferma: 'Esse in questi paesi sono più forti e attive degli uomini'. ${ }^{74}$ Successivamente, il 30 giugno, evidenzia una parità tra i sessi annotando: il paesetto di Ludvika 'deserto nei giorni feriali, perché tutti occupati, uomini e donne, nelle officine'. ${ }^{75}$ Il 2 luglio a Grangesberg, i ruoli rientrano nei limiti tradizionali: 'Qui le mogli seguono i mariti nei loro viaggi scientifici e dilettevoli, e lasciano i bambini in custodia alle donne di servizio le quali, non v'è dubbio, disimpegnano con scrupolosa coscienza il loro ufficio'. ${ }^{76}$

Pfeiffer, che sovente si sofferma sulle descrizioni dell'abbigliamento, non sviluppa affinità con le donne locali, anzi sembra che i piani interpretativi si ribaltino: da osservatrice diventa, con sommo fastidio, l'osservata:

I was, it appeared, quite a novel phenomenon in the eyes of these good people, and so they came one and all and stared at me; the women and children were, in particular, most unpleasantly familiar; they felt my dress, and the little ones laid their dirty little countenances in my lap. Added to this, the confined atmosphere from the number of persons present, their lamentable want of cleanliness, and their filthy habit of spitting, [etc.], all combined to form a most dreadful whole. ${ }^{77}$

Tale inversione di ruoli si consolida nella disattesa delle aspettative dei locali: 'I could not but wonder at the fact that most of these people expected to find me acquainted with a number of things generally studied only by men; they seemed to have a notion that in foreign parts women should be as learned as men'. ${ }^{78}$

Alec-Tweedie ripropone l'emancipazione della donna passando dalla libertà di potersi muovere, fare sport e relazionarsi con l'ambiente. Rievocando le intime e reciproche connessioni spazio-individuo, approdando nella 'terra incognita to most Europeans, viz., "Iceland", 79 non rifugge dal notare:

The first thing that struck us on landing was the sad, dejected look of the men and women who surrounded us. There was neither life nor interest depicted on their faces, nothing but stolid indifference. This apathy is no doubt caused by the hard lives these people live, the intense cold they have to endure, and the absence of variety in their every-day existence. What a contrast their faces afforded to the bright colouring and smiling looks one meets with in the sunny South. 80

Analogamente, netto è il giudizio sui Lapponi di Cappelli che li descrive come 'piccoli e brutti' e, aggiunge: '[...] per natura tristi e diffidenti, e non osammo rivolger loro la parola per timore di ricevere un mal garbo' ${ }^{81}$ Più ci si spinge al Nord, più gli individui sembrano incupirsi e contrastare esteticamente con il paesaggio naturale che li circonda. Entrambe le autrici sono tanto generose nelle loro descrizioni naturali quanto apparentemente inclementi o meramente schiette nel racconto delle popolazioni locali. Tali posizioni sono supportate da Pfeiffer che con piglio riporta:

I think, indeed, that the Icelanders are second to no nation in uncleanliness; not even to the Greenlanders, Esquimaux, or Laplanders. If I were to describe a portion only of what I experienced, my readers would think me guilty of gross exaggeration; I prefer, therefore, to

\footnotetext{
74 Ivi, p. 87.

75 Ivi, p. 111.

76 Ivi, p. 127.

77 Pfeiffer, Visit to Iceland, cit., p. 91.

78 Ivi, p. 91.

${ }^{79}$ Alec-Tweedie, A Girl's Ride, cit., p. 29.

80 Ivi, pp. 31-32.

${ }^{81}$ Cappelli, In Svezia, cit., p. 165.
} 
leave it to their imagination; merely saying that they cannot conceive any thing too dirty for Iceland delicacy. ${ }^{82}$

Pfeiffer nuovamente solletica la fantasia del lettore lasciando all'immaginazione il ruolo di colmare la mancanza testuale che la scrittrice evita di riportare in forma esplicita.

\section{Conclusioni}

Nel presente saggio si è tentato di evidenziare spaesamenti concreti derivanti da discordanze percettive, di matrice socio-culturale e geografica e di genere, che tipicamente s'intrecciano alla realtà fattuale nella narrazione odeporica, e straniamenti sia letterari, rintracciabili nelle scelte semantiche e stilistiche - che si identificano nell'offuscamento dei confini spazio-temporali (ridimensionando con opportune scelte semantiche la percezione degli oggetti, degli ambienti e degli individui) nonché nel capovolgimento dei piani d'osservazione - sia esistenziali, manifestazione dell'essere 'straniero' - riflessi nell'estetica descrittiva e nello scarto percepito tra realtà e aspettative. Il rapporto tra viaggio e scrittura s'innerva, infatti, su un'asse d'intima interdipendenza poiché in entrambi i processi di movimento, puramente fisico ma anche intimistico e narrativo, si produce un atto di spaesamento che si riverbera in uno straniamento letterario. In questa cornice, si ritiene, dunque, che lo spaesamento sia da intendersi come una condizione 'al di fuori' o di distonia con la realtà transitoria mentre lo straniamento sia la riformulazione di tale percezione, che a sua volta, conduce a nuove interpretazioni dell'io e dell'Alterità. Lo straniamento, riflesso nell'uso del linguaggio, negli intenti descrittivi e prospettici, che rendono l'oggetto insolito, si configura come cifra del mondo e la scrittura dello spaesamento diventa un varco di comprensione della realtà evenemenziale per un vasto pubblico eterogeneo.

Allontanatesi dalla dimensione conosciuta e familiare per confrontarsi con l'Altrove e l'Altro in un continuo incontro-scontro, nonostante i pregiudizi e le prescrizioni che gravano sulle donne che decidevano di mettersi in viaggio sole o per mete desuete, le quattro viaggiatrici prese in considerazione hanno affrontato in modo piuttosto simile la motilità che le ha portate nel Nord europeo giungendo, tuttavia, a interpretazioni e soluzioni dissimili. Le autrici, nella classica ermeneutica dello spaesamento che produce divergenze sostanziali tra realtà e immaginato, contribuiscono attivamente alla decostruzione e ricostruzione di stereotipi culturali tra i comuni lettori: ammantate di connotazioni positive sono indubbiamente le prospettive di Elisa Cappelli e, in un certo qual modo, le interpretazioni di Carla Serena, che parte però da un punto d'osservazione privilegiato; meno elogiative, più critiche e dirette, invece, le posizioni di Pfeiffer e Alec-Tweedie, quest'ultima particolarmente interessata agli aspetti scientifici.

Tanto mondana e ricca di personalità è la seppur breve trattazione di Carla Serena, quanto bucolica, pastorale, popolata da individui dalla peculiare 'bellezza nordica' (biondi, forti, silenziosi e rispettosi) è la Svezia di Elisa Cappelli. Lo sguardo dell'osservatrice italiana ridimensiona spazi, oggetti e persone, presentandoli in una luce spesso priva di storture, in linea, si ritiene, con gli intenti del volume, ossia di fornire uno strumento interpretativo ai discenti italiani. Dissimile è l'approccio di Pfeiffer, concorde con Alec-Tweedie: entrambe offrono un resoconto in accordo con i canoni del tempo arricchiti dall'intertestualità. Pfeiffer, in particolare, lascia al lettore il compito di colmare delle lacune immaginative; nel rovesciamento, in alcuni

\footnotetext{
82 Pfeiffer, Visit to Iceland, cit., p. 125.
} 
casi, dei piani interpretativi si rintracciano quei semi brechtiani di straniamento che consentono al lettore di sviluppare una prospettiva critica.

Parole chiave

diari di viaggio, spaesamento, straniamento, Cappelli, Pfeiffer, Alec-Tweedie, Carla Serena

Ellen Patat è attualmente docente a contratto presso il Dipartimento di Lingue e Letterature Straniere dell'Università degli Studi di Milano, SSML “P.M. Loria” e Università degli Studi Bicocca. Dopo la laurea in Lingue e Letterature Straniere, ha conseguito il dottorato di ricerca in Plurilinguismo e Letterature Comparate presso l'Università degli Studi di Udine (Italia), un Master in Interpretazione di conferenza $(E M C l)$ presso l'Università Boğaziçi (Istanbul,Turchia) e una seconda laurea in Lingua e cultura italiana per stranieri. I suoi studi riguardano la letteratura di viaggio moderna e contemporanea, la didattica EAP e ESP, e la scienza della traduzione. Tra le sue recenti pubblicazioni si ricordano: “I "bellicosi” paesaggi anni '80. Le cronache di viaggio di Gianni Celati e Guido Ceronetti', in: Quaderns d'Italià, 24 (2019), pp. 113126; 'Migrating identities. The Italian Female Gaze: Around the World in Thirty-four Stories', in: In Passage: The International Journal of Writing and Mobility, 1,1 (2018), pp. 1-16; 'L'identita' femminile - personale, sociale e globale - in Love in a Headscraf di Janmohamed e The Caged Virgin di Hirsi Ali', in: Le Simplegadi, XVI, 18 (2018), pp. 82-97.

Università degli Studi di Milano

Facoltà di Studi Umanistici

Dipartimento di Lingue e letterature straniere

Piazza S. Alessandro 1

20123 - Milano (Italia)

ellen.patat@unimi.it

\section{SUMMARY}

\section{The displacement of European female Travellers in the North}

\section{Estrangements in travel writing}

The present paper focuses on the travel accounts written by four women who decided to visit the European North in mid- and late-Nineteenth century: Ida Laura Pfeiffer's Visit to Iceland and Scandinavian North (1853), Carla Serena's Mon voyage personnels: souvenirs De la Baltique à la Mer Caspienne (1881), Ethel Brianna Tweedies' A girl's ride in Iceland (1889), and Elisa Cappelli's In Svezia. Impressioni di viaggio (1902). The aim is to analyse these travel diaries to identify the various forms of displacement and estrangement presented to the readers. The term 'displacement' is here to be understood as the condition of the 'outsider', the perception of being 'the Other', whereas 'estrangement' could be considered the reformulation of this awareness. This paper highlights real displacements that derive from social, cultural, geographical, and gender discrepancies adopting a comparative approach, which concentrates on the textual and semantic solutions, also taking into account the interdependence of travel and writing and of space and people. These aspects intertwine with the factual reality of the travel discourse, ultimately leading to both 'literary' and 'existential' estrangements. 